OPEN ACCESS

Edited by:

Paolo Landini,

University of Milan, Italy

Reviewed by:

Adam Jorgensen,

Wake Forest Institute for Regenerative

Medicine, United States

Marco Rinaldo Oggioni,

University of Leicester,

United Kingdom

${ }^{*}$ Correspondence:

Makrina Totsika

makrina.totsika@qut.edu.edu;

makrina.totsika@qut.edu.au

Specialty section:

This article was submitted to

Antimicrobials, Resistance

and Chemotherapy,

a section of the journal

Frontiers in Microbiology

Received: 23 December 2019

Accepted: 24 March 2020

Published: 14 May 2020

Citation:

Bolle ECL, Verderosa $A D$,

Dhouib R, Parker TJ, Fraser JF,

Dargaville TR and Totsika M (2020) An

in vitro Reconstructed Human Skin

Equivalent Model to Study the Role

of Skin Integration Around

Percutaneous Devices Against

Bacterial Infection.

Front. Microbiol. 11:670.

doi: 10.3389/fmicb.2020.00670

\section{An in vitro Reconstructed Human Skin Equivalent Model to Study the Role of Skin Integration Around Percutaneous Devices Against Bacterial Infection}

Eleonore C. L. Bolle1,2,3, Anthony D. Verderosa ${ }^{3}$, Rabeb Dhouib ${ }^{3}$, Tony J. Parker', John F. Fraser', Tim R. Dargaville' ${ }^{1}$ and Makrina Totsika ${ }^{3 *}$

${ }_{1}^{1}$ Tissue Repair and Translational Physiology Program, Institute of Health and Biomedical Innovation, Queensland University of Technology, Brisbane, QLD, Australia, ${ }^{2}$ The Innovative Cardiovascular Engineering and Technology Laboratory, Critical Care Research Group, The Prince Charles Hospital, Brisbane, QLD, Australia, ${ }^{3}$ Infection and Immunity Research Program, Institute of Health and Biomedical Innovation, School of Biomedical Sciences, Faculty of Health, Queensland University of Technology, Brisbane, QLD, Australia

Percutaneous devices are a key technology in clinical practice, used to connect internal organs to external medical devices. Examples include prosthesis, catheters and electrical drivelines. Percutaneous devices breach the skin's natural barrier and create an entry point for pathogens, making device infections a widespread problem. Modification of the percutaneous implant surface to increase skin integration with the aim to reduce subsequent infection is attracting a great deal of attention. While novel surfaces have been tested in various in vitro models used to study skin integration around percutaneous devices, no skin model has been reported, for the study of bacterial infection around percutaneous devices. Here, we report the establishment of an in vitro human skin equivalent model for driveline infections caused by Staphylococcus aureus, the most common cause of driveline-related infections. Three types of mock drivelines manufactured using melt electrowriting (smooth or porous un-seeded and porous pre-seeded with human fibroblasts) were implanted in human skin constructs and challenged with $S$. aureus. Our results show a high and stable load of $S$. aureus in association with the skin surface and no signs of $S$. aureus-induced tissue damage. Furthermore, our results demonstrate that bacterial migration along the driveline surface occurs in micro-gaps caused by insufficient skin integration between the driveline and the surrounding skin consistent with clinical reports from explanted patient drivelines. Thus, the human skin-driveline infection model presented here provides a clinicallyrelevant and versatile experimental platform for testing novel device surfaces and infection therapeutics.

Keywords: Staphylococcus aureus, 3D skin models, tissue engineered skin, melt electrowriting, percutaneous implant, driveline infections 


\section{INTRODUCTION}

Percutaneous devices are routinely used in medicine to connect internal organs to external medical devices or their components. Examples include drivelines for the transmission of electrical currents to ventricular assist devices (VADs), urinary peritoneal and vascular catheters for the transfer of fluids, or bone protheses for the transfer of forces (von Recum, 1984). VADs are a type of mechanical circulatory support used in heart failure therapy. They are attached to the heart and assist the heart in generating sufficient output to ensure adequate end organ perfusion (Koval and Stosor, 2019). VADs are powered and controlled through an external battery pack and controller unit. The connection between the pump and the battery pack and controller unit is maintained via a driveline, which contains insulated wires to carry current and telemetric data (Feldmann et al., 2018). In the United States alone, a minimum of 2,500 patients are added to the patient registry for mechanical circulatory support in any given year (Kirklin et al., 2017). Percutaneous devices, such as drivelines, cross the skin through a surgical incision and disrupt the skin's natural barrier creating an entry point for pathogens (von Recum, 1984). Thus, infections around percutaneous devices are highly prevalent with recent studies reporting catheter infection rates to be in the range of $2-33 \%$ over the life of the device, depending on the type of catheter (Salwiczek et al., 2014).

Driveline infections, in particular, account for $83 \%$ of ventricular assist device-specific infections, with incidence rates of 1.31 and 1.42 driveline infections per 100 patient months at 3 month pre- and post-device implantation, respectively (INTERMACS, 2015). More importantly, driveline infections are associated with an impaired clinical outcome, with patient survival rates only $76 \%$ by 24 month after the first driveline infection (Hannan et al., 2019). Driveline infections are primarily caused by bacteria, with fungal infections also reported less frequently (Hannan et al., 2019; Koval and Stosor, 2019). Grampositive bacteria from the Staphylococcus genus are the most common causative agents, with Staphylococcus aureus accounting for $28-44 \%$ of all driveline infections (Koval and Stosor, 2019).

The most common drivelines currently in clinical practice are made from a smooth material (silicone or polyurethane, depending on the manufacturer) and surrounded by a porous poly(ethylene terephthalate) sleeve, commercialized as Dacron ${ }^{\mathrm{TM}}$. The purpose of the Dacron ${ }^{\mathrm{TM}}$ sleeve is to increase tissue integration and prevent pathogen entry. While manufacturer guidelines recommend that the Dacron ${ }^{\mathrm{TM}}$ sleeve should be implanted so that it interfaces with the skin at the exit site, recent studies have demonstrated significantly reduced infection rates with the Dacron ${ }^{\mathrm{TM}}$ implanted subcutaneously and the smooth section of the driveline crossing the skin instead (Singh et al., 2012; Dean et al., 2015; McCandless et al., 2015; Camboni et al., 2016).

Despite clinical findings demonstrating reduced infection rates when smooth driveline sections cross the skin, there is evidence that smooth surfaces do not allow for sufficient skin integration, thus leading to the formation of a sinus tract around the implant (Winter, 1974; von Recum and Park, 1981;
Isackson et al., 2011). A sinus tract is formed by the epidermal layer migrating downwards parallel to the device, in an attempt to restore epidermal continuity (von Recum, 1984). The epidermal downwards migration can be reduced by increasing skin integration into the implant through the inclusion of pores to the surface of the percutaneous implant (Winter, 1974; Fukano et al., 2010). The prevailing opinion in the field of percutaneous devices is that increased skin and tissue integration will in turn create a tighter biological seal at the device exist site against invading pathogens and thus decrease infection rates (von Recum, 1984; Tagusari et al., 1998; Choi et al., 1999; Fukano et al., 2010; Isackson et al., 2011).

We have previously shown that porous scaffolds manufactured by melt electrowriting (MEW), an additive manufacturing technique, supports fibroblasts growth (Farrugia et al., 2013) and increases skin integration compared to smooth tubes in a 3D reconstructed human skin equivalent (HSE) model. Moreover, pre-seeding the porous scaffolds with human dermal fibroblasts prior to device implantation in HSEs appeared to reduce epidermal downgrowth, compared to un-seeded porous implants (Bolle et al., 2019). Based on these findings, the aim of the present study was to investigate whether increased skin integration around MEW porous implants can create a better biological seal against pathogen entry. Using a reconstructed HSE model, this is the first study to investigate $S$. aureus driveline-specific infections and provides a versatile in vitro platform for the detailed investigation of factors contributing to this significant clinical challenge worldwide.

\section{MATERIALS AND METHODS}

\section{Keratinocyte and Fibroblast Cell Isolation and Culture}

Keratinocytes were isolated from human skin samples obtained from patients undergoing abdominoplasty surgery and breast reduction surgery (Xie et al., 2010) and cultured according to previously published protocols (Rheinwald and Green, 1975; Dawson et al., 2006; Haridas et al., 2016). In brief, following an enzyme digestion overnight in $0.125 \%$ trypsin (Invitrogen, Australia) the epidermal layer was separated from the dermal layer. Keratinocytes were isolated from the epidermal layer and cultured on a layer of irradiated 3T3 fibroblasts in keratinocyte growth medium (Invitrogen, Australia). Fibroblasts were isolated from the dermis and cultured in fibroblasts growth medium, as previously described (Haridas et al., 2016).

\section{D Reconstructed Human Skin Equivalent Preparation and Culture}

The de-epidermised dermis (DED) was prepared following protocols described by Chakrabarty et al. (1999) and Dawson et al. (2006). The DEDs were between approximately $15 \times 15 \mathrm{~mm}$ and $20 \times 20 \mathrm{~mm}$ in size and were stored at $4^{\circ} \mathrm{C}$ in antibiotic/antimycotic medium consisting of DMEM supplemented with $1 \% \mathrm{v} / \mathrm{v}$ penicillin/streptomycin 
(Invitrogen). Skin constructs, referred to hereafter as HSEs, were generated as described previously (McGovern et al., 2013; Bolle et al., 2019). Briefly, $3.6 \times 10^{4}$ keratinocytes and $1.8 \times 10^{4}$ fibroblasts were seeded onto the DEDs through a stainless-steel ring with an inner diameter of $9 \mathrm{~mm}$ and lifted at the air liquid interface $48 \mathrm{~h}$ post seeding (Figure 1). HSEs were cultured in keratinocyte growth medium with fresh medium replaced every $48 \mathrm{~h}$. DEDs and cells were not patient-matched.

\section{Driveline Manufacture}

All mock (wireless, i.e., do not carry electrical charge) drivelines utilized in this study were manufactured from medical grade polymer poly( $\varepsilon$-caprolactone; PCL, Corbion Purac, Netherlands). PCL scaffolds were manufactured via MEW as previously described (Bolle et al., 2019) using an in-house custom built machine previously described in Wunner et al. (2017). Briefly, the PCL was loaded into a plastic syringe (Nordson EFD, United States), heated $\left(84^{\circ} \mathrm{C}\right)$ and extruded by applying air pressure. A voltage of $6.7 \mathrm{kV}$ was applied to the needle and the jet was collected on a grounded and motorized $x-$ $y$ collector plate in a lattice with layers at $90^{\circ}$ and $45^{\circ}$ to each other. The scaffolds were cut into $10 \times 10 \mathrm{~mm}$ squares using a scalpel.

To produce mock drivelines with different surfaces, a $4 \mathrm{~mm}$ stainless steel mandrel was dip-coated into a $10 \% \mathrm{w} / \mathrm{v}$ solution of PCL in chloroform, as previously described (Bolle et al., 2019).
The mandrel was then immersed in ethanol to aid in the removal of the PCL from the mandrel, resulting in smooth, hollow PCL tubes, from now onward referred to as PCL drivelines. To obtain tubular scaffolds, the scaffolds were wrapped around the heated, PCL coated mandrels to fuse the bottom layers of the scaffolds to the PCL core. The PCL tube-scaffold constructs were removed from the mandrel with the aid of ethanol. This resulted in hollow tubes with a solid core and porous outer surface with an outer diameter of $4.5 \mathrm{~mm}$, referred to as unseeded drivelines from now onward. All drivelines were sterilized by immersion in $80 \%$ ethanol for $30 \mathrm{~min}$, dried in a laminar flow hood and exposed to UV light for $20 \mathrm{~min}$ on either side. The MEW scaffolds and the PCL tube - scaffold constructs (porous mock drivelines) are shown in Figure 2. Scanning electron microscopy (SEM) images were acquired using a Zeiss Sigma Field Emission SEM, equipped with a Zeiss Gemini column at an accelerating voltage of $5 \mathrm{kV}$. All samples were gold sputter coated prior to imaging using a Leica EM-SCD005. Stereomicroscope images of the samples were acquired on a Nikon SMZ25 stereomicroscope (Nikon, Japan).

\section{Pre-seeding of Porous Mock Drivelines With Human Fibroblasts}

The tubular scaffolds were seeded with fibroblasts as previously described (Bolle et al., 2019). Briefly, the scaffolds were placed in 12-well plates following an overnight incubation

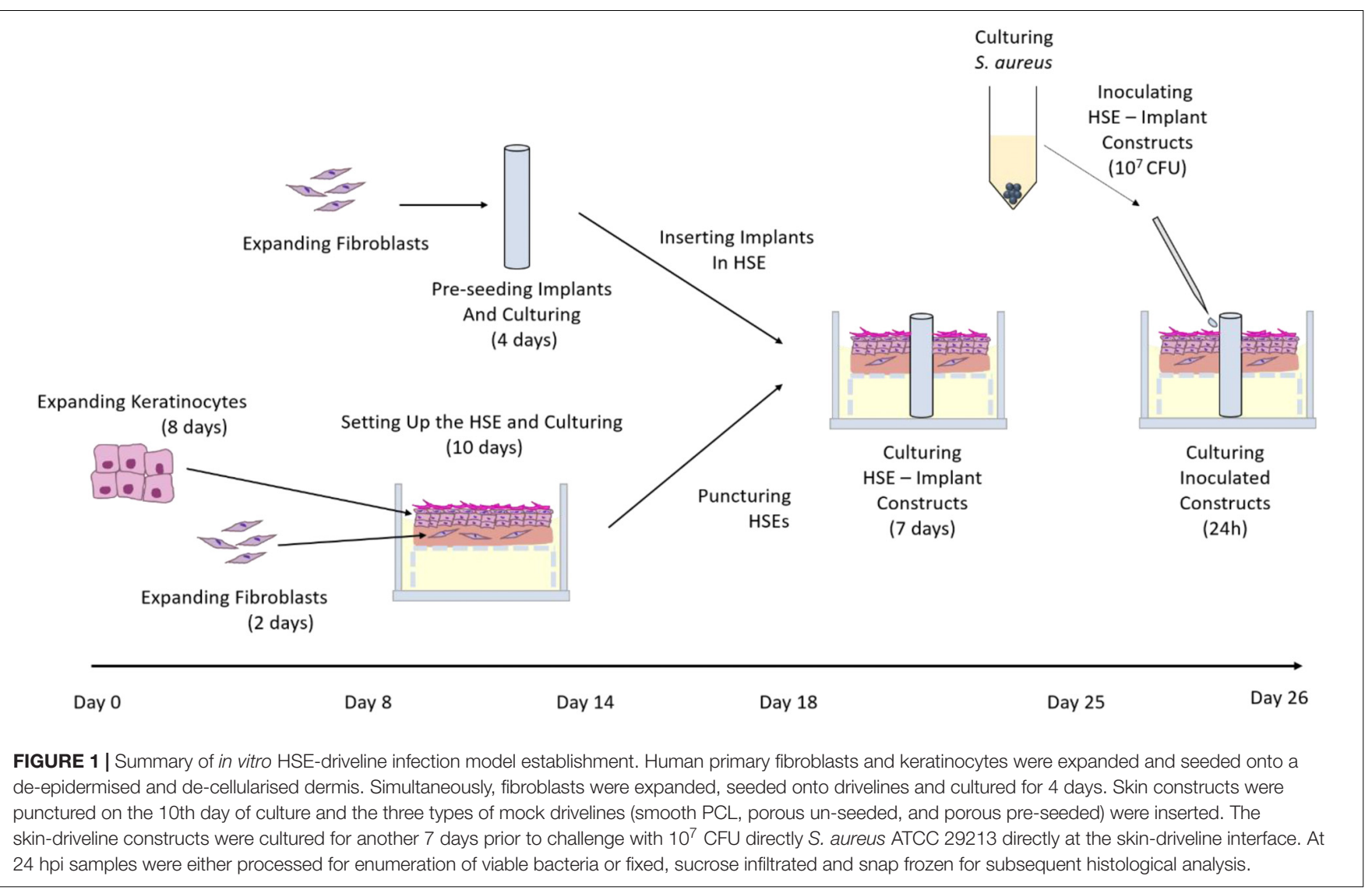


in fibroblasts growth medium and seeded with human dermal fibroblasts isolated according to the above described protocol, by applying $30 \mu \mathrm{L}$ containing $2 \times 10^{5}$ cells onto the scaffold. Following $30 \mathrm{~min}$ of incubation to allow for initial cell attachment, the scaffolds were inverted and another $30 \mu \mathrm{L}$ containing $2 \times 10^{5}$ cells were placed on the scaffolds. Following a further $30 \mathrm{~min}$ incubation the samples were submerged in fibroblasts growth medium and cultured for 4 days, with medium changed every $48 \mathrm{~h}$. The pre-seeded scaffolds are hereafter referred to as pre-seeded drivelines.

\section{Implanting Human Skin Equivalents With Mock Drivelines}

The mock drivelines were inserted into HSEs 10 day post seeding of fibroblasts and keratinocytes, as previously described (Bolle et al., 2019). Briefly, $4 \mathrm{~mm}$ holes were created in the HSEs with a biopsy punch (Stiefel) and the mock drivelines inserted from the dermal side and carefully pulled through using forceps (Figure 1). Skin-driveline constructs were cultured at the air liquid interface with medium replaced every $48 \mathrm{~h}$.

\section{Bacterial Strains and Culture Conditions}

Staphylococcus aureus ATCC 29213 was used in this study and routinely cultured in Lysogeny broth (LB) medium with shaking (200 RPM) at $37^{\circ} \mathrm{C}$. For HSE inoculations, bacterial cultures were collected at $16 \mathrm{~h}$, centrifuged and resuspended in antibiotic-free keratinocyte growth medium at a cell density of $5 \times 10^{8}$ colony forming units $(\mathrm{CFU}) / \mathrm{mL}$. An inoculum volume of $20 \mu \mathrm{L}\left(1 \times 10^{7} \mathrm{CFU}\right)$ was used in all HSE infection assays.

\section{S. aureus Infection Assay of Human Skin Equivalents Implanted With Mock Drivelines}

Human skin equivalent culture medium was replaced with antibiotic-free keratinocyte growth medium $72 \mathrm{~h}$ prior to infection. Intact HSEs controls (without a driveline) were inoculated by depositing $1 \times 10^{7}$ CFU S. aureus ATCC 29213 in a small $20 \mu \mathrm{L}$ volume at the centre of the construct surface. HSEs with implants were infected by depositing $4 \times 5 \mu \mathrm{L}$ of $S$. aureus inoculum in a cross-shape around the tubular implant interface. The cross-shaped pattern was chosen to ensure that the inoculum volume was evenly distributed around the entire circumference of the mock drivelines. Inoculated HSEs were incubated in antibiotic-free keratinocyte growth medium at $5 \% \mathrm{CO}_{2}$ and $37^{\circ} \mathrm{C}$. At $24 \mathrm{~h}$ post inoculation (hpi), HSEs were processed for histology or for viable CFU counts as described in sections below. Time-matched uninfected HSEs served as controls. A schematic overview of the entire experimental timeline is shown in Figure 1.

\section{Enumeration of Viable S. aureus From Infected Human Skin Equivalents}

Human skin equivalents collected at 24 hpi were dissected (roughly $3 \times 3 \mathrm{~mm}$ pieces) under aseptic conditions, weighed and digested in a $1 \mathrm{mg} / \mathrm{mL}$ collagenase solution in phosphate buffered saline (PBS; Gibco, Australia) at $37^{\circ} \mathrm{C}$ for $3 \mathrm{~h}$ in a shaking incubator (200 RPM). The samples were then homogenized mechanically in a mini-Beadbeater (Daintree Scientific, Australia) with stainless steel beads $(0.5 \mathrm{~mm}$, Daintree Scientific). Samples were homogenized for $15 \mathrm{~min}$ in $2 \mathrm{~min}$ cycles at 2,500 RPM, with 1 min rest on ice between cycles. HSE homogenates were then serially diluted 10 -fold in PBS and viable
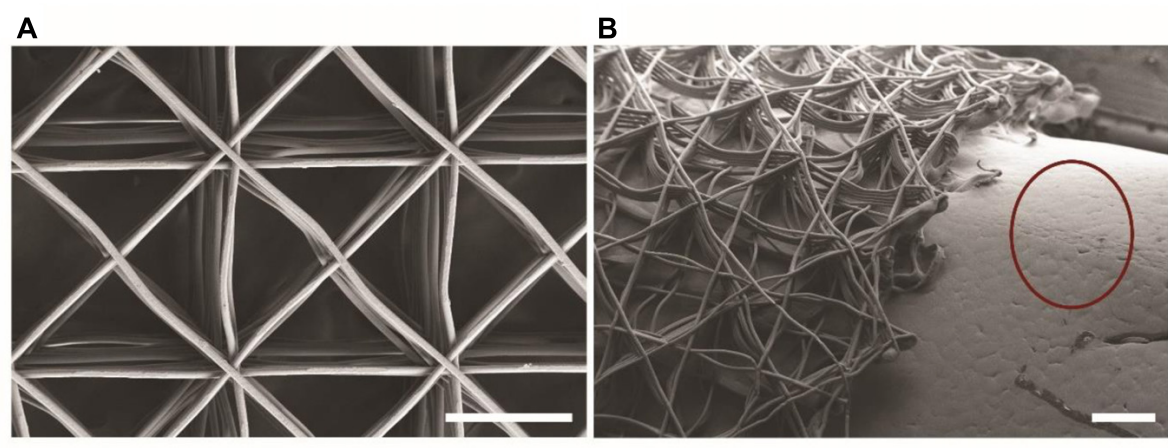

C

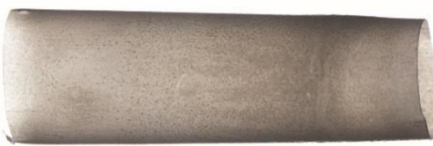

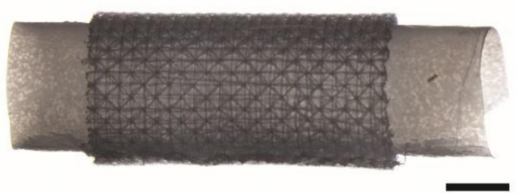

FIGURE 2 | Mock drivelines manufactured by melt electrowriting (MEW). SEM images of (A) a MEW scaffold used for constructing porous mock drivelines and (B) a porous mock driveline showing the MEW scaffold fused to the PCL tube (scale bars: $300 \mu \mathrm{m}$ ). The red circle highlights the surface morphology of the smooth PCL tube. Stereomicroscope images of (C) a smooth PCL tube and (D) a porous driveline (MEW scaffold - PCL tube) construct. Scale bars: 2 mm. 
bacteria (CFU) in samples were enumerated by plating triplicate $5 \mu \mathrm{L}$ aliquots of each dilution onto LB agar followed by overnight incubation at $37^{\circ} \mathrm{C}$.

\section{Histological Examination of S. aureus Infected Human Skin Equivalents}

Intact HSEs and HSEs with mock drivelines collected at $24 \mathrm{hpi}$ were prepared for histological analysis as described in Bolle et al. (2019). Briefly, the HSEs were rinsed in PBS supplemented with magnesium and calcium and fixed in 4\% PFA for a minimum of $30 \mathrm{~min}$. HSEs were then infiltrated in a sucroseOCT solution (optimal cutting temperature, Tissue Tek, Finland) with increasing ratios of OCT to preserve tissue morphology. HSEs were then snap frozen in a dry ice-ethanol slush and stored at $-80^{\circ} \mathrm{C}$ until required for further processing. Frozen HSE samples were sectioned at $20 \mu \mathrm{m}$ thickness on a cryostat (Leica Biosystems) and stained with hematoxylin and eosin (Bolle et al., 2019). Coverslips were applied using prolong gold mounting medium (Life Technologies) and slides were imaged using a Zeiss Axio Imager M2 microscope (Zeiss) or a 3D Histech Scan II Brightfield Slide Scanner (3D Histech, Hungary). For localizing $S$. aureus within the tissue, HSE sections were stained following a published Gram staining protocol, with modifications (Becerra et al., 2016). Briefly, the sections were rinsed, stained with crystal violet for $1 \mathrm{~min}$, incubated with iodine for $2 \mathrm{~min}$, decolorised in acetone and counterstained with safranin for $1 \mathrm{~min}$ (ProSciTech, Australia) with a wash performed between each step. Slides were imaged on a Leica DM2500 microscope (Leica, Germany).

\section{Fluorescence Microscopy of Explanted Mock Drivelines and S. aureus Staining}

Drivelines were carefully removed from HSEs using forceps immediately after collection at $24 \mathrm{hpi}$ and prepared for imaging of $S$. aureus colonization. Live/dead staining of $S$. aureus was performed using the LIVE/DEAD ${ }^{\mathrm{TM}}$ BacLight $^{\mathrm{TM}}$ Bacterial Viability kit L7007 (Life Technologies, Australia). Explanted drivelines were rinsed in saline $(0.9 \%)$ for $30 \mathrm{~s}$, then incubated in the dark at $30^{\circ} \mathrm{C}$ for $25 \mathrm{~min}$, with SYTO9 and Propidium Iodide (PI; $200 \mu \mathrm{L}$ ). The final applied concentration of SYTO9 and PI was $3.35 \mu \mathrm{M}$ and $20 \mu \mathrm{M}$, respectively. Following staining the drivelines were rinsed in saline $(0.9 \%)$ for $30 \mathrm{~s}$, mounted onto coverslips using ProLong ${ }^{\circledR}$ Diamond Antifade Mountant (Life Technologies, Australia) and immediately imaged on a Zeiss AxioVert A1 FL-LED microscope (Zeiss, Germany) utilizing a $100 \times$ oil immersion objective. Images were analyzed using the instrument software (Zen 2.3).

\section{Statistical Methods}

DEDs from 3 different donors were used for all experiments reported herein. In each experiment, DEDs from the same donor were tested in triplicate for each group (intact, PCL, un-seeded, pre-seeded). A total of 8 samples per group were analyzed by histology and a minimum of 6 for bacterial viability counts. CFU were enumerated by plating on agar plates and presented as CFU per $\mathrm{ml}$ of HSE homogenate. CFU from technical repeats were averaged and the average of each biological repeat is presented in dot plots per group. Group medians were compared for statistical differences by the non-parametric Kruskal Wallis test.

\section{RESULTS}

\section{Establishment of an in vitro Human Skin Equivalent Driveline Infection Model}

Staphylococcus aureus is the leading cause of driveline infections (Koval and Stosor, 2019) and has been previously shown to remain confined to the outermost layer of the epidermis in an HSE wound infection model (Shepherd et al., 2009). This makes it an ideal organism for studying driveline-specific infections in HSEs in vitro as it does not invade into deeper tissue, unlike the Gram-negative wound pathogen Pseudomonas aeruginosa (Shepherd et al., 2009), ensuring that any bacterial penetration can only occur via the driveline opening. To confirm this in our model, we used reference $S$. aureus ATCC 29213 cultures to inoculate intact HSEs, devoid of a driveline, and HSEs with a PCL driveline mimicking the smooth surface of those currently used in clinical practice. S. aureus was recovered at $24 \mathrm{~h}$ post inoculation (hpi) in similar numbers from both HSE groups, with an average of $1.2 \times 10^{7} \mathrm{CFU} / \mathrm{mL}$ recovered from intact HSEs and $1.3 \times 10^{7} \mathrm{CFU} / \mathrm{mL}$ from HSEs implanted with the PCL driveline (Figure 3A). As the number of bacteria recovered at 24 hpi was similar to the number of inoculated bacteria, this suggests that $S$. aureus ATCC 29213 remained viable in our experimental model over this time-period maintaining a high tissue load.

While bacterial numbers recovered from tissues were similar, the localization of $S$. aureus differed between HSE groups (Figures 3B, 4). Gram staining of cross sections from intact HSEs revealed that $S$. aureus cells were exclusively present in the apical surface of the skin, with bacteria loosely associated with the uppermost layer and no bacteria present in deeper epidermal layers (Figure 3B). Similar analysis of HSEs implanted with PCL drivelines (Figure 4) revealed heavy S. aureus colonization at the driveline-skin exit site (inoculation site), with minimal bacterial presence observed further down this interface. Where bacteria were seen to be present deeper into the skin-driveline interface, they appeared to be mostly found in areas where gaps were present between HSE tissue and driveline (Figure 4).

H\&E staining confirmed that all HSEs displayed a tissue morphology closely resembling that of native human skin (Figures 3B, 4). The epidermal growth patterns along the smooth PCL drivelines varied, with 5 out of 8 samples exhibiting epidermal downgrowth around the implant and 3 showing epidermal upgrowth. This is in line with our previous findings using smooth PCL drivelines in HSEs (Bolle et al., 2019) and suggests that the presence of $S$. aureus does not affect epidermal growth patterns. 
A

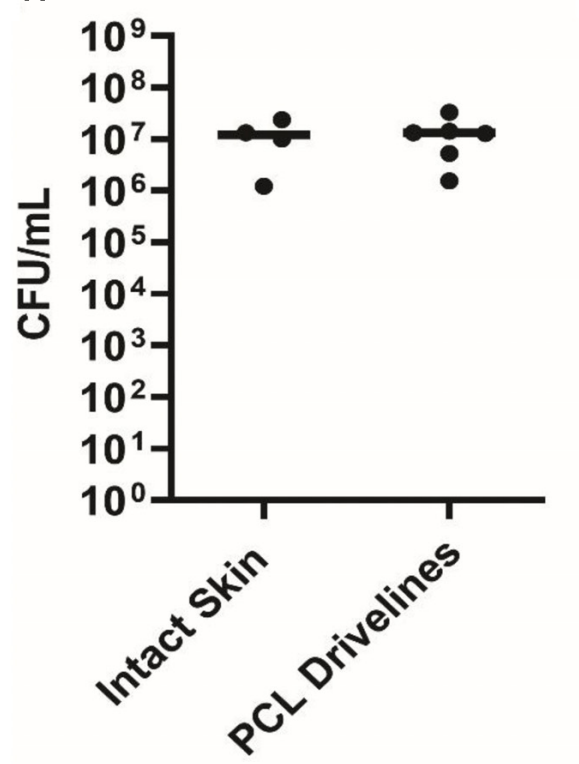

B
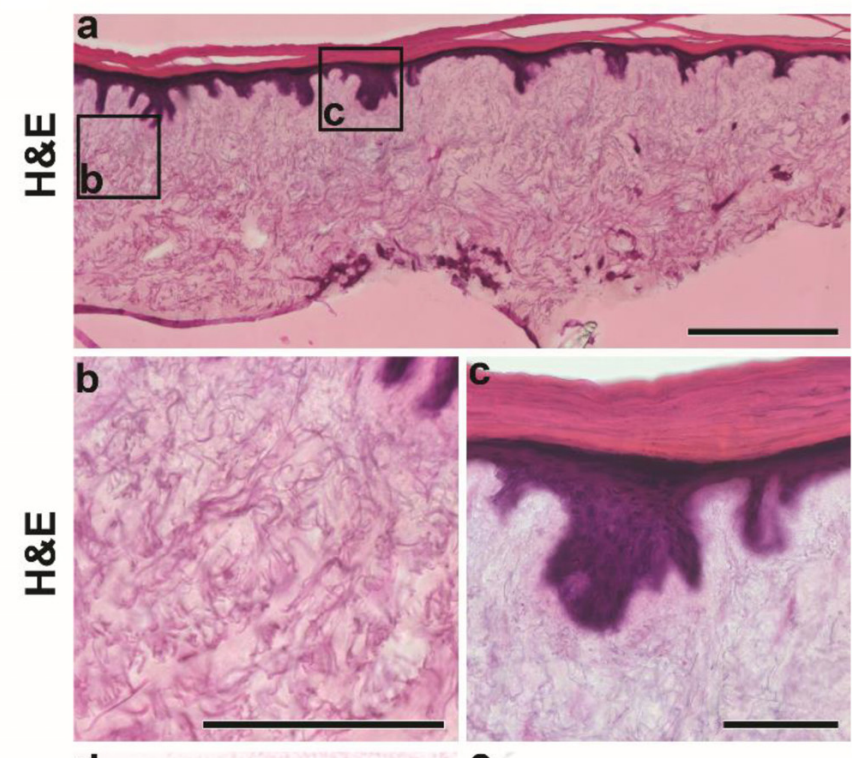

d

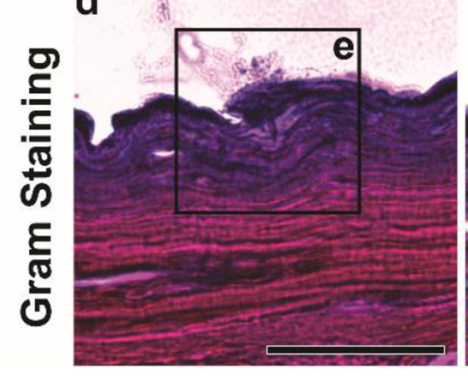

e

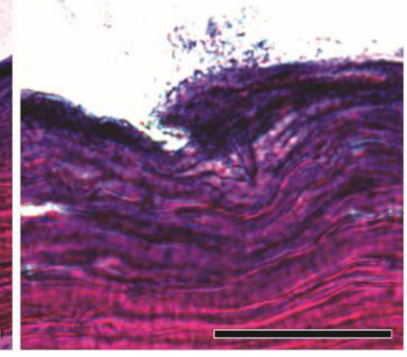

FIGURE 3 | Staphylococcus aureus infection of intact HSEs. (A) Number of viable S. aureus colony forming units (CFU) recovered from intact skin HSEs (no driveline) or HSEs implanted with smooth PCL drivelines at $24 \mathrm{~h}$ post inoculation. Bacterial numbers are presented as CFU/mL with group means shown as horizontal lines. Dot plots show data from a minimum of 4 experimental repeats using HSEs derived from 3 skin donors. (B) Histological analysis of infected intact HSEs showing an overview of cross sections of the entire skin construct by H\&E staining. Visible features include rete ridges between the epidermis and dermis (panel a, scale bar: $500 \mu \mathrm{m}$ ), connective dermal tissue (panel b, scale bar: $200 \mu \mathrm{m}$ ) and the epidermis and stratum corneum (panel c, scale bar: $100 \mu \mathrm{m}$ ). Gram staining of tissue cross-sections revealed $S$. aureus localizing exclusively on the uppermost layer of the epidermis in loose association with the skin tissue (panels d and e, scale bars: $100 \mu \mathrm{m}$ and $50 \mu \mathrm{m}$, respectively).

Collectively, our microbiological and histological analyses suggest that the HSE model is able to sustain a high bacterial load without $S$. aureus-induced tissue damage or invasion of deeper epidermal and dermal layers. Bacterial non-invasiveness was considered a pre-requisite for an HSE-driveline infection model which sought to establish bacterial migration along the opening created by the presence of a driveline. Further, the results with the PCL drivelines suggest that the presence of the driveline does not affect total bacterial counts and that most bacteria are localized at the driveline exit site or associated with the driveline surface as a biofilm. We observed little $S$. aureus migration along the PCL implant interface and only where there were micro-gaps present, similar to recent clinical observations from explanted patient drivelines (Qu et al., 2019a). Thus, we conclude that our established in vitro HSE-driveline infection model is a suitable platform for investigating differences in the biological seal created by different driveline surfaces, which we investigated next.

\section{S. aureus Forms a Biofilm at the Entry Site of Porous Drivelines and Migrates Along the Driveline-Tissue Interface via Micro-Gaps}

To test whether skin integration around different porous driveline surfaces translates to differences in bacterial migration along the driveline, we inoculated HSEs with $S$. aureus as above, but this time the HSEs were implanted with MEW un-seeded and pre-seeded mock drivelines. The $S$. aureus tissue load recovered at $24 \mathrm{hpi}$ was $3 \times 10^{6} \mathrm{CFU} / \mathrm{mL}$ and $8 \times 10^{6} \mathrm{CFU} / \mathrm{mL}$, for the un-seeded and pre-seeded groups, respectively (Figure 5A). Histological staining revealed infected HSEs with un-seeded and pre-seeded drivelines to have an intact dermis and epidermis, with the lateral side of the preseeded drivelines exhibiting an intact thin layer of fibroblasts covering the scaffolds (Figure 5B- top right panel). All HSEs bearing un-seeded implants ( 8 out of 8 ) displayed epidermal 


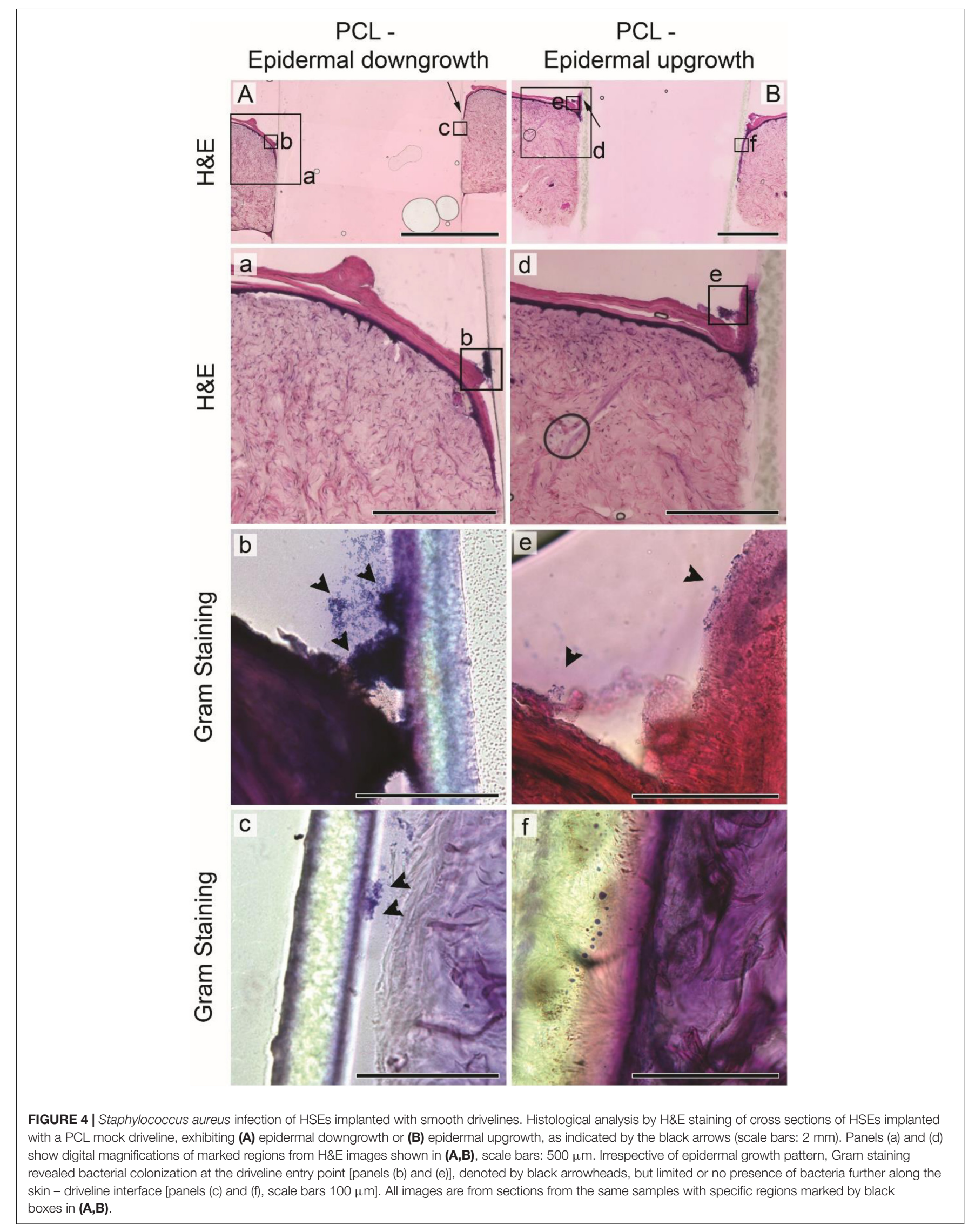



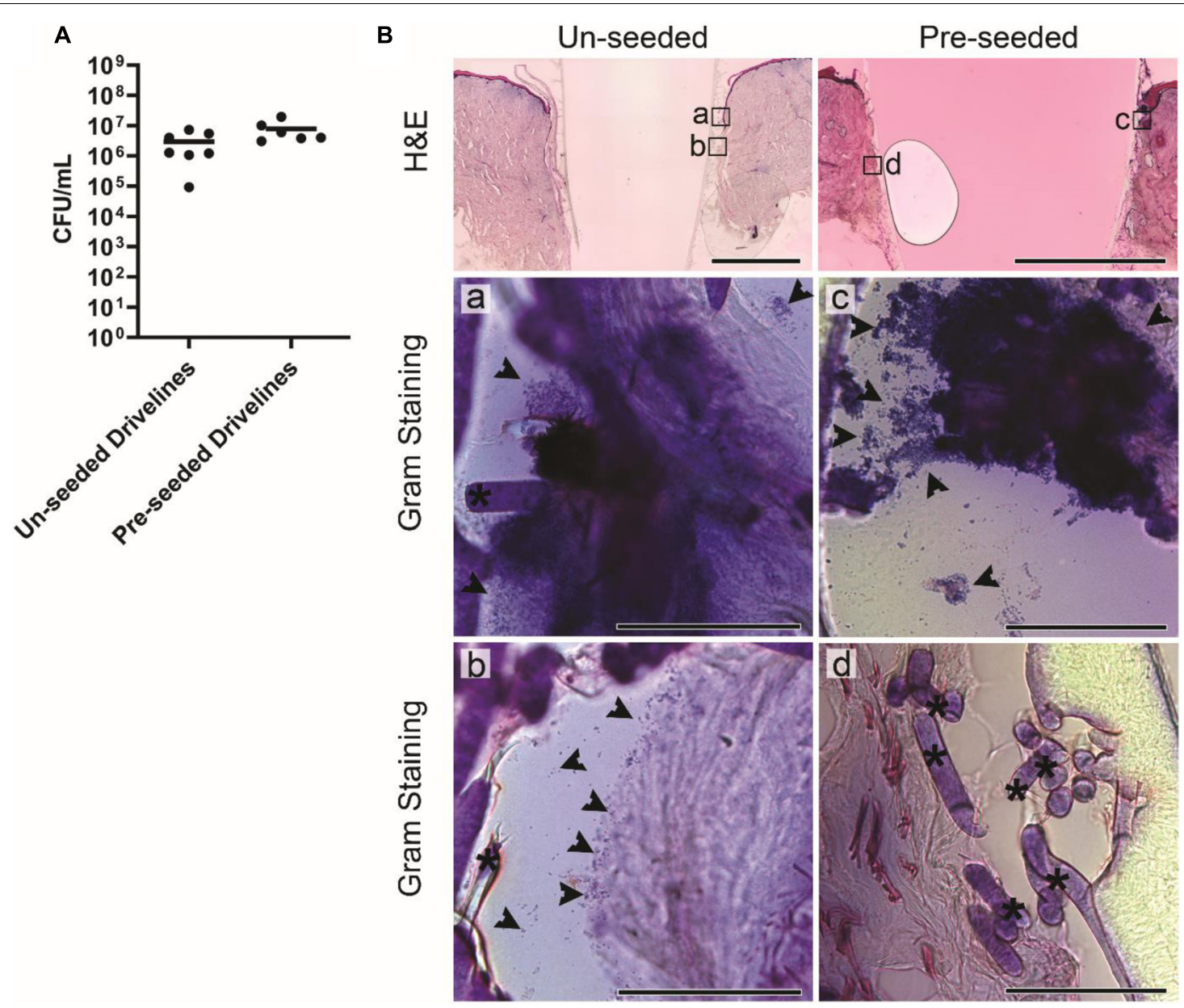

FIGURE 5 | Staphylococcus aureus infection of HSEs implanted with porous drivelines. (A) Number of viable S. aureus recovered from HSEs with un-seeded or pre-seeded drivelines at $24 \mathrm{hpi}$. Bacterial numbers are presented as CFU/mL with group means shown as horizontal lines. Dot plots show data from a minimum of 6 experimental repeats using HSEs derived from 3 donors. (B) Histological analysis of infected HSEs implanted with un-seeded and pre-seeded drivelines retained an intact epidermis and dermis as shown by H\&E staining of cross-sections (top panels; scale bars denote 2 mm). In HSEs with un-seeded drivelines, S. aureus (indicated by black arrows) localized at the inoculation site (a), with bacteria detected further along the interface and associated with deeper HSE tissue or present in gaps between the tissue and un-seeded driveline (b; Gram staining, left panel; scale bars denote $100 \mu \mathrm{m}$ ). In HSEs with pre-seeded drivelines, S. aureus localized at the inoculation site (c), but no bacterial colonization of the tissue in close contact with the driveline was observed (d; Gram staining, right panels; scale bars: $100 \mu \mathrm{m})$. Black asterisks denote PCL fiber struts.

downgrowth, but epidermal growth patterns around pre-seeded implants varied, with 4 exhibiting epidermal upgrowth and 4 epidermal downgrowth.

Staphylococcus aureus colonization of the driveline-skin entry site was invariantly observed in both HSE groups but was only noticeable further along the driveline-HSE interface where gaps were present between the driveline and the surrounding HSE tissue. Only small $S$. aureus clusters were observed to be associated with the deeper dermis interface, and only in HSEs implanted with un-seeded drivelines (Figure 5B), as these contained more gaps with the HSE tissue compared to preseeded or PCL drivelines. Similar to what was observed for PCL implants, S. aureus had formed a biofilm on the porous drivelines at the inoculation site, as revealed by LIVE/DEAD staining of explanted un-seeded and pre-seeded drivelines (Figure 6). Fluorescence microscopy along the entire length of the porous drivelines revealed $S$. aureus presence within the voids of the scaffold and along its fiber struts. S. aureus colonization and migration appeared to be more pronounced on the un-seeded drivelines, compared to pre-seeded PCL drivelines (Figure 6).

Taken together, our results suggest that epidermal growth pattern differences do not appear to strictly dictate whether $S$. aureus cells could migrate down the HSE-implant interface. Bacterial presence along the driveline surface was mostly associated with the presence of gaps along the tissue-driveline interface, which occurred more frequently in un-seeded porous drivelines. Moreover, challenging the skin-driveline interface with a high bacterial load did not impact the preestablished epidermal growth patterns at the implant entry site or the deeper dermal-implant interface, irrespective of the implant's surface. 


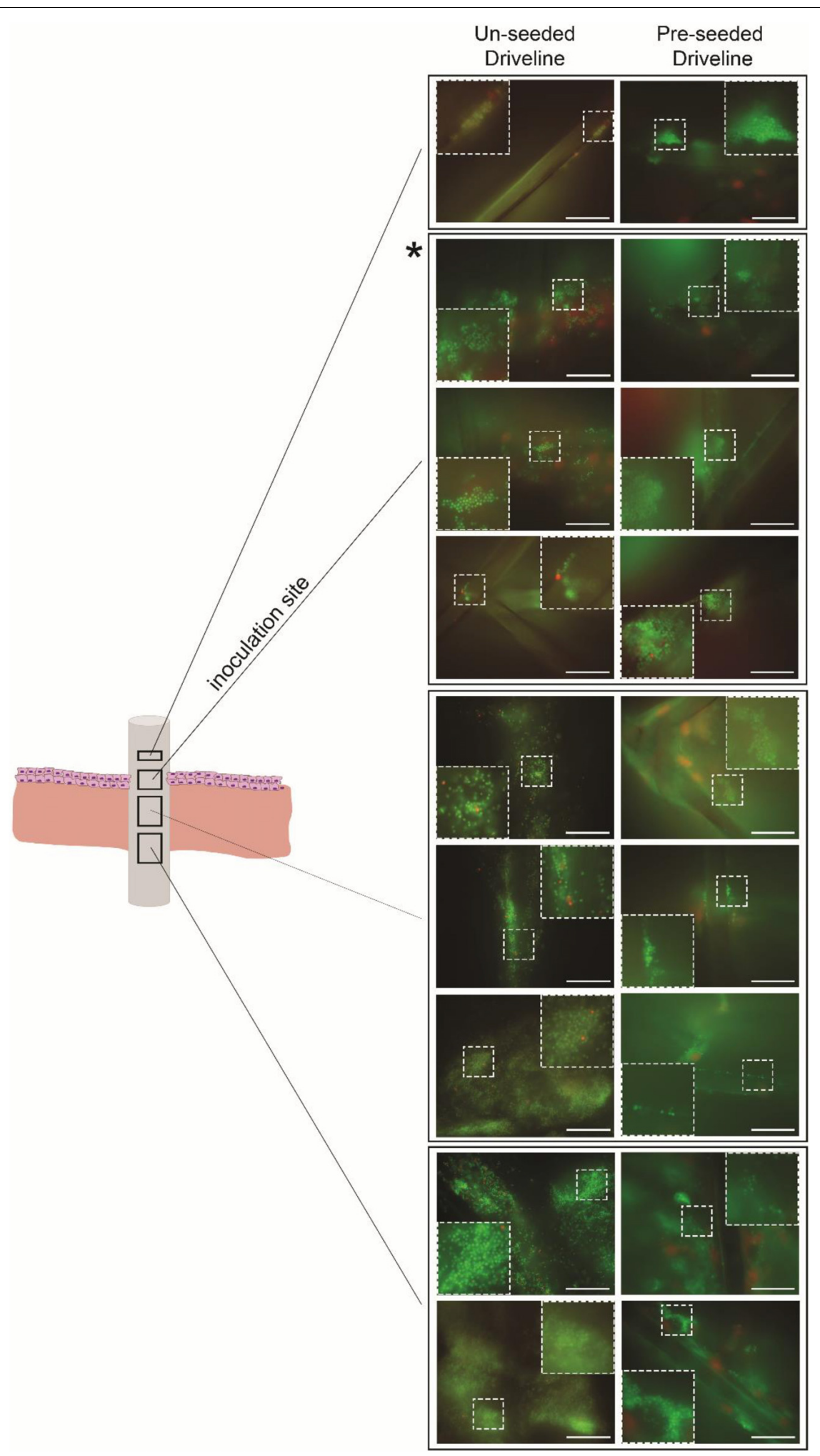

FIGURE 6 | Fluorescence microscopy of infected HSE-explanted drivelines. S. aureus cells were stained with bacterial LIVE/DEAD staining (live = green, dead = red). Live S. aureus cells (seen as green cocci) were present in the voids and on the fiber struts of the un-seeded and pre-seeded drivelines at the inoculation site (second quadrant from top, denoted by asterisk) and deeper down the driveline surface (left and right panels, respectively; Scale bars: $30 \mu \mathrm{m})$. Inserts show a digitally zoomed section (25\%) of the region marked by the white dotted line. 


\section{DISCUSSION}

Infections around percutaneous implants and drivelines are a prevalent clinical problem, since the advent of the earliest VADs, limiting successful outcomes of ventricular assist device therapy (Hannan et al., 2019). It has been hypothesized by us and others that increased skin and tissue integration around the percutaneous device translates to a better biological seal against bacteria, with the potential to reduce bacterial infection rates (von Recum, 1984; Tagusari et al., 1998; Choi et al., 1999; Fukano et al., 2010; Isackson et al., 2011; Bolle et al., 2019). The aim of this study was thus to investigate whether porous scaffolds that were previously shown to increase skin integration and device stability in an HSE model would create a protective biological seal against pathogen entry. This aim necessitated the establishment of the first HSE-driveline infection model for in vitro investigations.

In vitro skin models have greatly advanced the field of cutaneous research and are based on excised full thickness skin, or tissue engineered skin with the dermal component based on synthetic matrices or de-cellularised dermis (Abd et al., 2016; Pupovac et al., 2018). The de-cellularised dermis skin model (DED-HSE) is well characterized, closely resembles native human skin and has been shown to re-epithelialise (Topping et al., 2006; Xie et al., 2010; Fernandez et al., 2014). More importantly, it has been used to study bacterial infections of wounds and the efficacy of functionalized biomaterials in reducing the bacterial burden in infected skin (Shepherd et al., 2009, 2011; Zheng et al., 2019).

In vitro skin models, however, have not yet been utilized to study bacterial infections around drivelines. Current knowledge on driveline infections is mainly derived from in vitro agar or drip flow bioreactor models, ex vivo analysis of explanted drivelines or in vivo rodent models (Arrecubieta et al., 2009; Toba et al., 2011; Qu et al., 2019b,a). The in vitro agar model was established by Qu et al. (2019b) to mimic microbial biofilm growth on drivelines within the subcutaneous tissue tunnel. The model consists of casting a Hinton or Roswell Park Memorial Institute medium agar mold by placing a piece of smooth driveline into molten agar and removing the driveline upon setting of the agar. Following insertion of an inoculated piece of smooth or porous driveline, the tunnel is covered with a lid from the same agar. To mimic the driveline exit site, the authors use a drip-flow biofilm reactor, whereby they place inoculated pieces of smooth or porous driveline into the incubation chamber while pumping growth medium through the system (Qu et al., 2019b). While these agar models offer suitable in vitro models to study biofilm formation and bacterial migration in the subcutaneous tissue, they do not allow examination of epidermal and dermal integration or its association with bacterial migration. Ex vivo clinical samples, on the other hand, are highly pertinent to studying various aspects of driveline infections, but observations tend to be subject to biological variation, demanding analyses of large numbers of clinical samples, and as such do not lend themselves as an ideal experimental platform to validate hypotheses. Small animal models have been utilized to study the in vivo role of bacterial virulence factors in driveline colonization and infection, such as the SdrF surface protein of S. aureus (Arrecubieta et al., 2009) and more generally the role of biofilm formation on driveline infections by S. aureus and S. epidermidis (Toba et al., 2011). None of these studies, however, directly investigated the role of skin integration and epidermal growth patterns in protecting from bacterial infection, so the clinical value of rodent driveline infection models to study skin integration remains unknown.

To address the lack of physiologically relevant in vitro models to study the correlation between skin integration and bacterial infection around percutaneous drivelines, we established a firstin-field in vitro driveline infection model based on the established DED-HSE model. Our model reproduces the clinical features observed in explanted drivelines from infected patients and provides a suitable tool for the investigation of skin-driveline integration properties on infection outcome. To the best of our knowledge, this is the first study to directly test whether increased skin integration around porous drivelines translates to an increased biological seal against pathogen entry.

Our study utilized $S$. aureus as the model pathogen, given $S$. aureus is the most common causative agent of driveline infections (Koval and Stosor, 2019). We deliberately inoculated the driveline exit-site with a relatively high bacterial burden $\left(10^{7} \mathrm{CFU}\right.$ of $S$. aureus deposited in a small volume directly at the exit site), to challenge the biological seal created by the different driveline surfaces. Despite the high challenge, histological analysis confirmed that a high bacterial load could be maintained without tissue damage even up to $72 \mathrm{~h}$ post inoculation (data not shown). The non-invasiveness of $S$. aureus strains in HSE models, previously reported (Shepherd et al., 2009) and confirmed in this study for reference strain ATCC 29213, was important in ensuring that bacterial entry and migration occurred via the driveline and not via the tissue.

Our model also reproduced the formation of a sinus tract as a result of epidermal downgrowth around the implanted driveline. Sinus tract formation is a well-known mode of failure of percutaneous devices (von Recum, 1984) and has been hypothesized to create an environment conducive to bacterial proliferation, leading to subsequent device infection (Isackson et al., 2011; Affeld et al., 2012). Our study offers the first in vitro validation of this hypothesis, providing evidence of increased bacterial localization in the sinus tract between the driveline and the surrounding tissue. While in our model epidermal upgrowth was also observed, epidermal downgrowth was more frequently associated with implants that did not display tight integration with the surrounding HSE tissue and contained frequent micro-gaps along the length of the interface where bacteria were commonly observed. A recent analysis of infected drivelines explanted from patients undergoing heart transplants revealed insufficient integration between the Dacron ${ }^{\mathrm{TM}}$ and the surrounding subcutaneous tissue with bacterial presence within similar voids (Qu et al., 2019a). In an in vitro study by the same group, challenge of porous Dacron ${ }^{\mathrm{TM}}$ with the same $S$. aureus strain used in our study, also resulted in biofilm formation within the inter-fiber space of the intricate porous Dacron ${ }^{\mathrm{TM}}$ structure (Qu et al., 2019b), similar to what we observed with the scaffold voids of mainly un-seeded and some pre-seeded drivelines. These results indicate that a healthy and well-integrated drivelineinterface is crucial to reduce bacterial colonization and prevent migration along the implant interface. When the cell viability 
of the fibroblast layer forming the tight interface in our preseeded driveline-HSEs declined at $72 \mathrm{hpi}$, we observed increased bacterial migration into the driveline, further supporting this tenet (data not shown).

While there appears to be a consensus from in vitro and ex vivo studies on bacterial migration along porous drivelines, findings from animal models seem to be conflicting. A study by Isackson et al. (2011) in rabbits investigated four different types of titanium implants, all consisting of a percutaneous post and a subcutaneous anchor, with a smooth, porous or mixed surface (porous percutaneous post and smooth anchor or vice versa). Implants were repeatedly challenged with high-doses of $S$. aureus 4 weeks post implantation until manifestation of Grade II clinical signs of infection (experimental end point). Histological analysis revealed epidermal downgrowth and sinus tract formation in all implants, but implants with smooth components had a 7 -fold increased risk of infection compared to implants with a porous surface in one or both components (Isackson et al., 2011). It is noteworthy that while the authors confirmed $S$. aureus to be the only organism isolated from infected implants, bacterial loads from implants or tissues were not determined and localization of bacteria along the implant interface was not examined either. Similarly, older studies in rabbits and pigs reported no signs of infection with porous carbon percutaneous devices (Krouskop et al., 1988; Nowicki et al., 1990). Differences in implant materials could account for these differences in results, as well as the use of different bacterial pathogens, which are known to have distinct biofilm formation properties.

Perhaps the most obvious difference, however, between in vitro and in vivo studies, is the absence of immune cells from most in vitro human skin models. Less than a handful of reconstructed HSE models containing immune cells have been reported to date (Bechetoille et al., 2007; Ouwehand et al., 2011; Linde et al., 2012; Kühbacher et al., 2017), which is testament to the complexity of establishing and maintaining 3D co-culture models (Pupovac et al., 2018). Overcoming those limitations in the near future will greatly enhance the clinical value of in vitro HSE-based models, including our HSE-driveline infection model, given the primary role of immune cells in fighting invading pathogens. In addition, the model could be further enhanced by adding movement of the driveline in the HSE through a bioreactor, in order to mimic driveline movement caused by patient breathing and other motion. While in this study we aimed to compare the biological seal created by different porous driveline surfaces (un-seeded and pre-seeded), which were previously shown to promote different skin integration patterns (Bolle et al., 2019), our model lends itself nicely for other types of studies, e.g., comparative efficacy of different driveline materials (Dacron, silicone, etc.) or testing of new and existing antibacterials and disinfectants. Furthermore, the model allows investigation of host-pathogen interactions and could be useful for in vitro studies of bacterial factors contributing to skin and/or driveline colonization and translocation. While, in our study we chose to explore bacterial localization in HSE tissues and drivelines by staining and microscopy -analyses which allow bacterial localization but generate image data not optimal for quantitative measurements-, quantification of viable bacteria in the tissue (as done in our study) could be combined with enumeration of bacteria on the HSE-explanted drivelines (as done in other studies; Qu et al., 2019b,a), to provide total numbers of bacteria colonizing the entire model system. Bacterial migration through the driveline could also be quantified by enumerating viable CFU recovered from media surrounding the HSE in each well (Figure 1), but we would highly recommend using transwells for such investigations to exclude bacteria "spill-over" from the epidermal inoculation site, especially for small-sized HSE constructs (Bolle and Totsika, unpublished observations).

Despite certain limitations, the in vitro percutaneous driveline infection model reported herein represents a versatile tool to further our understanding of factors leading to driveline infections and the role of skin integration and driveline surface in reducing bacterial infections. Such models can also provide a valuable early preclinical platform for the testing of new antimicrobial surfaces and therapeutics aimed at reducing the prevalence of device-related infections burdening health systems worldwide.

\section{DATA AVAILABILITY STATEMENT}

The datasets generated for this study are available on request to the corresponding author.

\section{ETHICS STATEMENT}

The studies involving human participants were reviewed and approved by the Queensland University of Technology Research Ethics Committee (1300000063) and Uniting Healthcare/St Andrew's Hospital Ethics Committee (0346). The patients/participants provided their written informed consent to participate in this study.

\section{AUTHOR CONTRIBUTIONS}

EB and MT conceived the study and designed the experiments, analyzed and interpreted the data and wrote the manuscript. $\mathrm{EB}, \mathrm{AV}$, and RD conducted the experiments. JF, TP, and TD contributed to the design and implementation of the research. All authors revised the manuscript for content and approved the final version.

\section{FUNDING}

The authors would like to recognize the financial assistance provided by The Prince Charles Hospital Foundation (ER201514, PRO2014-08, EM2017-03, and TM2017-04), a Clive and Vera Ramaciotti Health Investment Grant (2017HIG0119 to MT), and the National Health and Medical Research Council Centre for Research Excellence (APP1079421) and Project Grant APP1144046. TD is supported by an ARC Future Fellowship (FT150100408) and MT by a QUT Vice-Chancellor's Research Fellowship. 


\section{ACKNOWLEDGMENTS}

Some of the data reported in this paper were obtained at the Infection and Immunity Ian Potter Foundation Imaging Suite operated by the Institute of Health and Biomedical Innovation

\section{REFERENCES}

Abd, E., Yousef, S. A., Pastore, M. N., Telaprolu, K., Mohammed, Y. H., Namjoshi, S., et al. (2016). Skin models for the testing of transdermal drugs. Clin. Pharmacol. Adv. Appl. 8, 163-176. doi: 10.2147/CPAA.S64788

Affeld, K., Grosshauser, J., Goubergrits, L., and Kertzscher, U. (2012). Percutaneous devices: a review of applications, problems and possible solutions. Expert Rev. Med. Devices 9, 389-399. doi: 10.1586/erd.12.25

Arrecubieta, C., Toba, F. A., Von Bayern, M., Akashi, H., Deng, M. C., Naka, Y., et al. (2009). SdrF, a Staphylococcus epidermidis surface protein, contributes to the initiation of ventricular assist device driveline-related infections. PLoS Pathog. 5:411. doi: 10.1371/journal.ppat.1000411

Becerra, S. C., Roy, D. C., Sanchez, C. J., Christy, R. J., and Burmeister, D. M. (2016). An optimized staining technique for the detection of Gram positive and Gram negative bacteria within tissue. BMC Res. Notes 9:216. doi: 10.1186/s13104-0161902-1900

Bechetoille, N., Dezutter-Dambuyant, C., Damour, O., André, V. R., Orly, I., and Perrier, E. (2007). Effects of solar ultraviolet radiation on engineered human skin equivalent containing both langerhans cells and dermal dendritic cells. Tissue Eng. 13, 2667-2679. doi: 10.1089/ten.2006.0405

Bolle, E. C. L., Bartnikowski, N., Haridas, P., Parker, T. J., Fraser, J. F., Gregory, S. D., et al. (2019). Improving skin integration around long-term percutaneous devices using fibrous scaffolds in a reconstructed human skin equivalent model. J. Biomed. Mater. Res. Part B Appl. Biomater. 108, 738-749. doi: 10.1002/jbm.b. 34428

Camboni, D., Zerdzitzki, M., Hirt, S., Tandler, R., Weyand, M., and Schmid, C. (2016). Reduction of INCOR\$driveline infection rate with silicone at the driveline exit site. Interact. Cardiovasc. Thorac. Surg. 24, 222-228. doi: 10.1093/ icvts/ivw336

Chakrabarty, K. H., Dawson, R. A., Harris, P., Layton, C., Babu, M., Gould, L., et al. (1999). Development of autologous human dermal-epidermal composites based on sterilized human allodermis for clinical use. Br. J. Dermatol. 141, 811-823. doi: 10.1046/j.1365-2133.1999.03153.x

Choi, L., Choudhri, A. F., Pillarisetty, V. G., Sampath, L. A., Caraos, L., Brunnert, S. R., et al. (1999). Development of an infection-resistant LVAD driveline: a novel approach to the prevention of device-related infections. J. Heart Lung Transplant. 18, 1103-1110. doi: 10.1016/s1053-2498(99)00076-5

Dawson, R. A., Upton, Z., Malda, J., and Harkin, D. G. (2006). Preparation of cultured skin for transplantation using insulin-like growth factor I in conjunction with insulin-like growth factor binding protein 5, epidermal growth factor, and vitronectin. Transplantation 81, 1668-1676. doi: 10.1097/01. tp.0000226060.51572.89

Dean, D., Kallel, F., Ewald, G. A., Tatooles, A., Sheridan, B. C., Brewer, R. J., et al. (2015). Reduction in driveline infection rates: results from the heartmate ii multicenter driveline silicone skin interface (SSI) registry. J. Hear. Lung Transplant. 34, 781-789. doi: 10.1016/j.healun.2014.11.021

Farrugia, B. L., Brown, T. D., Upton, Z., Hutmacher, D. W., Dalton, P. D., and Dargaville, T. R. (2013). Dermal fibroblast infiltration of poly( $\varepsilon$-caprolactone) scaffolds fabricated by melt electrospinning in a direct writing mode. Biofabrication 5:025001. doi: 10.1088/1758-5082/5/2/025001

Feldmann, C., Chatterjee, A., Haverich, A., and Schmitto, J. D. (2018). "Left ventricular assist devices - a state of the art review," in Heart Failure: From Research to Clinical Practice, Vol. 3, ed. M. S. Islam (Cham: Springer International Publishing), 287-294. doi: 10.1007/5584_2018_145

Fernandez, T. L., Van Lonkhuyzen, D. R., Dawson, R. A., Kimlin, M. G., and Upton, Z. (2014). Characterization of a human skin equivalent model to study the effects of ultraviolet b radiation on keratinocytes. Tissue Eng. Part C Methods 20, 588-598. doi: 10.1089/ten.tec.2013.0293

Fukano, Y., Usui, M. L., Underwood, R. A., Isenhath, S., Marshall, A. J., Hauch, K. D., et al. (2010). Epidermal and dermal integration into sphere-templated and at the Central Analytical Research Facility operated by the Institute for Future Environments (QUT). The authors thank the staff from the Central Analytical Research Facility for assistance with the histological staining and Dr. Shaun D. Gregory for help with initial conceptualization of this study.

porous poly(2-hydroxyethyl methacrylate) implants in mice. J. Biomed. Mater. Res. Part A 94A, 1172-1186. doi: 10.1002/jbm.a.32798

Hannan, M. M., Xie, R., Cowger, J., Schueler, S., de By, T., Dipchand, A. I., et al. (2019). Epidemiology of infection in mechanical circulatory support: a global analysis from the ISHLT mechanically assisted circulatory support registry. J. Heart Lung Transplant. 38, 364-373. doi: 10.1016/j.healun.2019. 01.007

Haridas, P., McGovern, J. A., Kashyap, A. S., McElwain, D. L. S., and Simpson, M. J. (2016). Standard melanoma-associated markers do not identify the MM127 metastatic melanoma cell line. Sci. Rep. 6:24569. doi: 10.1038/srep24569

INTERMACS (2015). Interagency registry for mechanically assisted circulatory support (INTERMACS) quarterly statistical report 2015 Q1. Statist. Rep. 126, 1401-1406. doi: 10.1161/circulationaha.112.097816

Isackson, D., McGill, L. D., and Bachus, K. N. (2011). Percutaneous implants with porous titanium dermal barriers: an in vivo evaluation of infection risk. Med. Eng. Phys. 33, 418-426. doi: 10.1016/j.medengphy.2010.11.007

Kirklin, J. K., Pagani, F. D., Kormos, R. L., Stevenson, L. W., Blume, E. D., Myers, S. L., et al. (2017). Eighth annual INTERMACS report: special focus on framing the impact of adverse events. J. Hear. Lung Transplant. 36, 1080-1086. doi: 10.1016/j.healun.2017.07.005

Koval, C. E., and Stosor, V. (2019). Ventricular assist device related infections and solid organ transplantation - guidelines from the american society of transplantation infectious diseases community of practice. Clin. Transplant. 33:e13529. doi: 10.1111/ctr.13529

Krouskop, T., Brown, H., Gray, K., Shively, J., Romovacek, G., Spira, M., et al. (1988). Bacterial challenge study of a porous carbon percutaneous implant. Biomaterials 9, 398-404. doi: 10.1016/0142-9612(88)90003-8

Kühbacher, A., Henkel, H., Stevens, P., Grumaz, C., Finkelmeier, D., BurgerKentischer, A., et al. (2017). Central role for dermal fibroblasts in skin model protection against Candida albicans. J. Infect. Dis. 215, 1742-1752. doi: 10.1093/ infdis/jix153

Linde, N., Gutschalk, C. M., Hoffmann, C., Yilmaz, D., and Mueller, M. M. (2012). Integrating macrophages into organotypic co-cultures: a $3 \mathrm{D}$ in vitro model to study tumor-associated macrophages. PLoS One 7:e40058. doi: 10.1371/journal. pone.0040058

McCandless, S. P., Ledford, I. D., Mason, N. O., Alharethi, R., Rasmusson, B. Y., Budge, D., et al. (2015). Comparing velour versus silicone interfaces at the driveline exit site of HeartMate II devices: infection rates, histopathology, and ultrastructural aspects. Cardiovasc. Pathol. 24, 71-75. doi: 10.1016/j.carpath. 2014.07.011

McGovern, J. A., Heinemann, J. R., Burke, L. J., Dawson, R., Parker, T. J., Upton, Z., et al. (2013). Stratum basale keratinocyte expression of the cell-surface glycoprotein CDCP1 during epidermogenesis and its role in keratinocyte migration. Br. J. Dermatol. 168, 496-503. doi: 10.1111/bjd.12119

Nowicki, B., Runyan, R. S., Smith, N., and Krouskop, T. A. (1990). Kinetics of colonization of a porous vitreous carbon percutaneous implant. Biomaterials 11, 389-392. doi: 10.1016/0142-9612(90)90092-90095

Ouwehand, K., Spiekstra, S. W., Waaijman, T., Scheper, R. J., de Gruijl, T. D., and Gibbs, S. (2011). Technical advance: langerhans cells derived from a human cell line in a full-thickness skin equivalent undergo allergen-induced maturation and migration. J. Leukoc. Biol. 90, 1027-1033. doi: 10.1189/jlb.0610374

Pupovac, A., Senturk, B., Griffoni, C., Maniura-Weber, K., Rottmar, M., and McArthur, S. L. (2018). Toward immunocompetent 3D skin models. Adv. Healthc. Mater. 7:1701405. doi: 10.1002/adhm.201701405

Qu, Y., McGiffin, D., Hayward, C., Robson, D., Kure, C., Thissen, H., et al. (2019a). A study of infected drivelines from ventricular assist device patients: the presence of microbial biofilms and micro-gaps in the driveline tunnel. J. Hear. Lung Transplant. 38, S102-S103. doi: 10.1016/j.healun.2019.01.240

Qu, Y., McGiffin, D. C., Kure, C. E., Ozcelik, B., Thissen, H., Fraser, J. F., et al. (2019b). Microbial biofilm formation and migration on ventricular assist device 
drivelines: implications for infection. J. Thorac. Cardiovasc. Surg. 37:S134. doi: 10.1016/J.HEALUN.2018.01.323

Rheinwald, J. G., and Green, H. (1975). Serial cultivation of strains of human epidemal keratinocytes: the formation keratinizin colonies from single cell. Cell 6, 331-343. doi: 10.1016/s0092-8674(75)80001-8

Salwiczek, M., Qu, Y., Gardiner, J., Strugnell, R. A., Lithgow, T., McLean, K. M., et al. (2014). Emerging rules for effective antimicrobial coatings. Trends Biotechnol. 32, 82-90. doi: 10.1016/j.tibtech.2013.09.008

Shepherd, J., Douglas, I., Rimmer, S., Swanson, L., and MacNeil, S. (2009). Development of three-dimensional tissue-engineered models of bacterial infected human skin wounds. Tissue Eng. Part C Methods 15, 475-484. doi: 10.1089/ten.tec.2008.0614

Shepherd, J., Sarker, P., Rimmer, S., Swanson, L., MacNeil, S., and Douglas, I. (2011). Hyperbranched poly(NIPAM) polymers modified with antibiotics for the reduction of bacterial burden in infected human tissue engineered skin. Biomaterials 32, 258-267. doi: 10.1016/j.biomaterials.2010.08.084

Singh, A., Russo, M. J., Valeroso, T. B., Johnson, E. M., Anderson, A. S., Fedson, S. E., et al. (2012). Modified HeartMate II driveline externalization technique significantly decreases incidence of infection and improves long-term survival. J. Hear. Lung Transplant. 31:S20. doi: 10.1016/j.healun.2012.01.035

Tagusari, O., Yamazaki, K., Litwak, P., Kojima, A., Klein, E. C., Antaki, J. F., et al. (1998). Fine trabecularized carbon: ideal material and texture for percutaneous device system of permanent left ventricular assist device. Artif. Organs 22, 481-487. doi: 10.1046/j.1525-1594.1998.06152.x

Toba, F. A., Akashi, H., Arrecubieta, C., and Lowy, F. D. (2011). Role of biofilm in Staphylococcus areus and Staphylococcus epidermis in ventricular assist device infection. J. Thorac. Cardiovasc. Surg. 141, 1259-1264. doi: 10.1016/j.jtcvs.2010. 07.016.Role

Topping, G., Malda, J., Dawson, R., and Upton, Z. (2006). Development and characterisation of human skin equivalents and their potential application as a burn wound model. J. Wound Manag. 14, 14-21. von Recum, A. F. (1984). Applications and failure modes of percutaneous devices: a review. J. Biomed. Mater. Res. 18, 323-336. doi: 10.1002/jbm.820180403

von Recum, A. F., and Park, J. B. (1981). Permanent percutaneous devices. Crit. Rev. Bioeng. 5, 37-77.

Winter, G. D. (1974). Transcutaneous implants: reactions of the skin? implant interface. J. Biomed. Mater. Res. 8, 99-113. doi: 10.1002/jbm.820080311

Wunner, F. M., Bas, O., Saidy, N. T., Dalton, P. D., Pardo, E. M. D.J., and Hutmacher, D. W. (2017). Melt electrospinning writing of threedimensional Poly( $\varepsilon$-caprolactone) scaffolds with controllable morphologies for tissue engineering applications. J. Vis. Exp. 45:e56289. doi: 10.3791/ 56289

Xie, Y., Rizzi, S. C., Dawson, R., Lynam, E., Richards, S., Leavesley, D. I., et al. (2010). Development of a three-dimensional human skin equivalent wound model for investigating novel wound healing therapies. Tissue Eng. Part C Methods 16, 1111-1123. doi: 10.1089/ten.TEC.2009.0725

Zheng, K., Balasubramanian, P., Paterson, T. E., Stein, R., MacNeil, S., Fiorilli, S., et al. (2019). Ag modified mesoporous bioactive glass nanoparticles for enhanced antibacterial activity in 3D infected skin model. Mater. Sci. Eng. C 103:109764. doi: 10.1016/j.msec.2019.109764

Conflict of Interest: The authors declare that the research was conducted in the absence of any commercial or financial relationships that could be construed as a potential conflict of interest.

Copyright (c) 2020 Bolle, Verderosa, Dhouib, Parker, Fraser, Dargaville and Totsika. This is an open-access article distributed under the terms of the Creative Commons Attribution License (CC BY). The use, distribution or reproduction in other forums is permitted, provided the original author(s) and the copyright owner(s) are credited and that the original publication in this journal is cited, in accordance with accepted academic practice. No use, distribution or reproduction is permitted which does not comply with these terms. 